\section{TRANSMISSION AVATARS}

The transmission majorly converts engine power into vehicle traction through other vehicle sub-systems. About six per cent of the vehicle energy loss is due to transmission and driveline systems. Besides fuelefficiency, it impacts ownership costs, carbon footprint, and driving experience and comfort. It is apparent that vehicles in different regions are segregated into manual and automatic transmissions. The mobility patterns and innovations have naturally evolved over regional preferences but now it is a mixed global deployment.

Transmissions are intelligent and through driver display interfaces either prompt the driver to shift to the next gear, lower or higher, or automatically shift into different gears in non-manual transmissions. Many vehicles also are designed to display upfront to the driver the predicted fuel economy that will be attained through the driver shift behaviour and patterns. Most transmissions are designed to provide a sporty mode, fuel economy mode, off-road and conventional on-road modes and the load hauling high traction mode.

\section{HOLISTIC INTEGRATION}

Transmission development has become a holistic integration of light weighting, gear ratio analyses, gear design, electronic controls, sensor technologies \& controls, clutch materials, design \& interfaces, torque converter variants, software interfaces in conventional and hybrid architectures, lubricants, lubrication techniques and thermal management.

The design and development of transmissions has undergone a radical change through virtual engineering tools and techniques ranging from CAE for stress analysis, thermal management, and dynamic loading, and for simulation and study of virtual shift quality behaviour, use of hardware and software in the loop tools and processes to emulate real-life driving conditions. Such tools and processes have remarkably reduced the development time and cost, and ensured innovations in better quality transmissions for a wider array of vehicle portfolios.

In manual transmissions, adding gears allows the engine to operate at a more efficient speed more often, and the more gearing options a vehicle has, the more fuel efficient it is (four to seven per cent). On the other hand, CVTs use a pair of variable-diameter pulleys connected by a belt or chain that can produce an infinite number of engine/ wheel speed ratios.

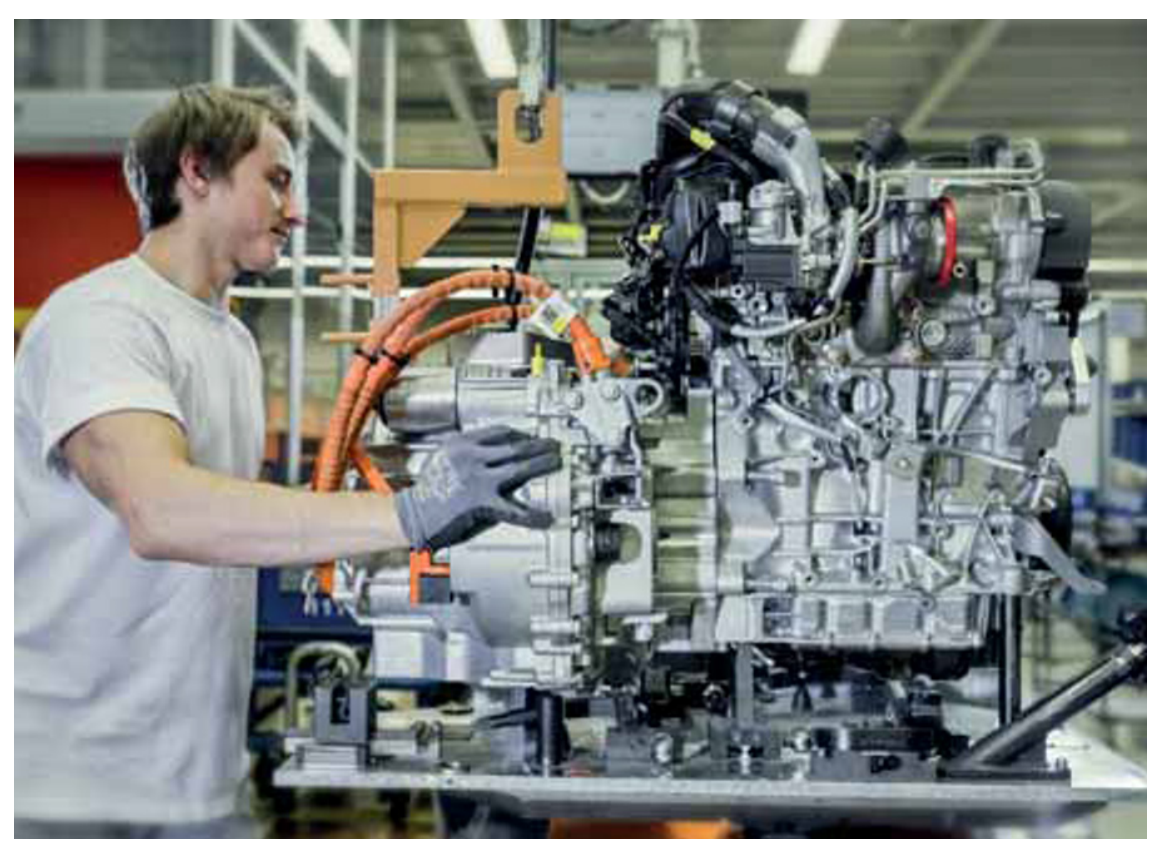

DCTs are four to five per cent more efficient than geared transmissions. They operate similarly to a manual transmission except that they do not require clutch actuation or shifting by the driver. Automatic shifting is controlled electronically (shift-by-wire) and performed by a hydraulic system or electric motor. In addition, more efficient, higher-speed automatic transmissions (eight to nine-speed) will be more popular in the near future.

The hybridised versions of transmissions are integrated and designed with pancake type electric motors that are sandwiched between the engine and transmission interface. The integration of electric motor introduces different types of complexity in terms of thermal management, lubrication, concentricity, bending loads, mechanical interfaces and the electrical outlets and shielding. With an optimised gear layout, bearings, main shaft, counter shaft and idler shafts orientation, the transmissions have become more compact and can accommodate the electric motor in hybrid versions with least impact on powertrain packaging.

In electric mobility or wheel motor driven hybrid vehicles, transmissions are almost non-existent. Though these were invented in late 19th century, they are getting popular now and are a cultural mindset change for mobility practitioners and the consumers. Eliminating the drivetrain, including gearboxes, differentials, drive shafts and axles, provides a significant weight and manufacturing cost saving, while also decreasing the environmental impact. These have added to the positives for a paradigm shift to zero emission mobility.

Innovation in transmissions has been driven by a confluence of factors that drive higher fuel efficiency and low carbon footprint to utilise technology for differentiation, while meeting increasingly stringent regulations. 